\title{
BLIND ON-LINE DIGITAL CALIBRATION OF MULTI-STAGE NYQUIST-RATE AND OVERSAMPLED A/D CONVERTERS
}

\author{
Gert Cauwenberghs \\ Department of Electrical and Computer Engineering \\ The Johns Hopkins University, Baltimore, MD 21218-2686 \\ E-mail: gert@bach.ece.jhu.edu
}

\begin{abstract}
A linear calibration technique for on-line digital correction of ana$\log$ imprecisions in the implementation of multi-stage analog-todigital converters, is presented. The calibration is 'blind', in that it uses no reference and operates directly on the digital output during conversion. The only assumption made on the input signal is that its spectrum is bandlimited. The excess sampling over Nyquist bandwidth is used for least-squares auto-zeroing calibration. The technique extends directly to digital correction of multi-stage oversampled A/D converters where a multi-bit quantizer is implemented using a pipelined algorithmic A/D converter. Behavioral simulations on pipelined algorithmic and dual-quantization oversampled A/D converters demonstrate significant improvements in signal-toquantization-noise performance over the uncalibrated case.
\end{abstract}

\section{INTRODUCTION}

Cascaded multi-stage designs for algorithmic and oversampled analog-to-digital conversion $[1,2]$, such as pipelined A/D converters [3], and multiple-quantization cascaded delta-sigma modulators $[4,5]$, offer efficient analog hardware implementations with optimal trade-off between resolution, speed and hardware complexity and power dissipation. The resolution that can be attained by these designs severely depends on precision and matching of analog components in the implementation, such as amplifier gain and capacitor ratios. To a large extent, the effect of analog circuit imperfections can be undone in the digital domain by postprocessing the output bit streams. Digital calibration techniques have been applied to multi-stage pipelined [3] and multiple-quantization oversampled $[6,7,8]$ A/D converters. On-line techniques offer the advantage of continuous adaptation in the background of the conversion process $[6,8]$. A nice example with efficient hardware implementation is given in [8] where the adaptation eliminates the quantization noise of a first quantizer along with a test signal injected externally into the quantizer, thereby minimally interferring with the input signal.

This paper introduces an on-line 'blind' calibration technique which does not require a reference signal, applicable to pipelined algorithmic A/D converters, multi-stage oversampled A/D converters and their combinations. The technique assumes a bandlimited input signal, reserving a stop band to correct for any linear errors in the analog implementation by autozeroing the output response

This work was partly supported by NSF Career Award MIP-9702346, and by Northrop Grumman and the Maryland Industrial Partnerships Program (MIPS).

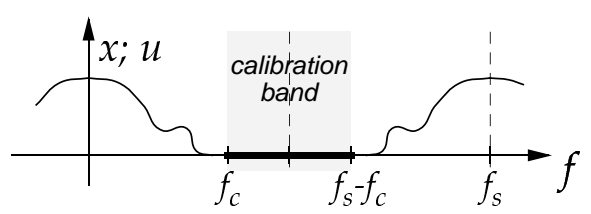

Figure 1: Signal stop band for blind calibration.

in the stop band through least-squares linear parameter estimation, as illustrated schematically in Figure 1.

Analog circuit models for algorithmic and oversampled data converters are summarized in the next section, with corresponding models for linear digital correction. The blind calibration technique is defined and applied to (super-)Nyquist-rate algorithmic A/D conversion in Section 3, extended to multi-stage oversampled A/D conversion in Section 4, and further discussed and concluded in Section 5.

\section{ANALOG MODELS AND DIGITAL CORRECTION}

The models are discrete-time with both analog and digital signals represented in the $z$-domain, the operator $z$ denoting a unit time advance. One time step corresponds to the reciprocal of the sampling frequency, $f_{s}$, which exceeds the Nyquist frequency $f_{N}$. Analog signals are normalized to the range $[-1,+1]$, and binary signals are \pm 1 .

\subsection{Multi-Stage Delta-Sigma Modulator}

Without loss of generality, we consider a dual-stage oversampled design of the Leslie-Singh [5] topology as depicted in Figure 2. A second-order delta-sigma modulator

$$
\begin{aligned}
z v & =\beta_{1} v+\alpha_{1}\left(u-q_{1}\right) \\
z w & =\beta_{2} w+\alpha_{2}\left(v-q_{1}\right)
\end{aligned}
$$

with input $u$ and one-bit quantized output

$$
q_{1}=Q_{1}(w)=\operatorname{sgn}(w)
$$

interfaces to a second stage with multi-bit quantized output

$$
q_{2}=Q_{2}(w)=w+e_{2}
$$

$e_{2}$ representing the quantization noise contributed by $Q_{2}$. The output bits from both stages are digitally combined

$$
q=F_{1}(z) q_{1}+F_{2}(z) q_{2}
$$




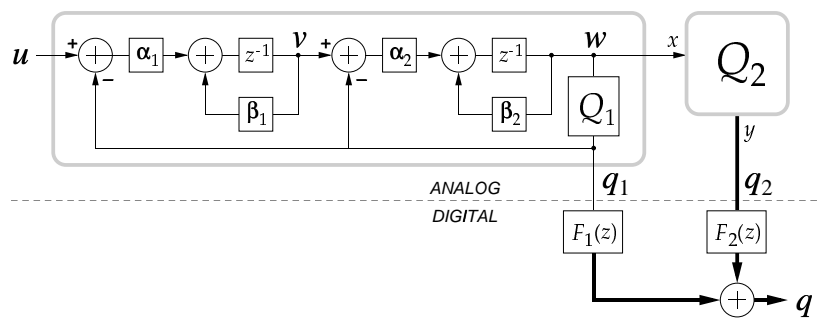

Figure 2: Multi-stage, multiple quantization oversampled $A / D$ conversion. Example topology with second-order $\Delta \Sigma$ modulator and multi-bit quantizer $Q_{2}$ (Leslie-Singh, 1990).

in order to eliminate the quantization noise introduced in the first stage, yielding second-order noise-shaping modulated, secondstage multi-bit quantization error

$$
q \equiv z^{-2} u-\frac{1-\beta_{1} z^{-1}}{\alpha_{1}} \frac{1-\beta_{2} z^{-1}}{\alpha_{2}} e_{2}
$$

obtained by the following choice of FIR filters

$$
\begin{aligned}
& F_{1}(z)=z^{-2}+z^{-1} \frac{1-\beta_{1} z^{-1}}{\alpha_{1}} \\
& F_{2}(z)=\frac{1-\beta_{1} z^{-1}}{\alpha_{1}} \frac{1-\beta_{2} z^{-1}}{\alpha_{2}}
\end{aligned}
$$

The key to digital correction is a technique to adaptively estimate these filter coefficients [7] or a suitable subset $[6,8]$. The results outlined below hold for any form of the second quantizer $Q_{2}$, which itself could implement a multi-stage oversampled modulator [4]. In what follows, we consider the special case where the second quantizer $Q_{2}$ is a pipelined algorithmic $\mathrm{A} / \mathrm{D}$ converter. In a practical implementation of this arrangement, the $\Delta \Sigma$ quantizer $Q_{1}$ shares the first stage (most significant bit) of $Q_{2}$ [5].

\subsection{Algorithmic A/D Conversion}

A radix- $\gamma$ bit-level sub-ranging data converter converts an input $x$ into $n$ output bits $y_{i}$ through algorithmic recursion of binary quantization and residues

$$
\begin{aligned}
y_{i} & =Q\left(x_{i-1}\right)=\operatorname{sgn}\left(x_{i-1}\right), \quad i=1 \ldots n \\
x_{i} & =\gamma_{i} x_{i-1}-\delta_{i} y_{i}+\epsilon_{i}
\end{aligned}
$$

starting from $x_{0} \equiv x$. For ideal radix-2 conversion, $\gamma_{i} \equiv 2, \delta_{i} \equiv 1$ and $\epsilon_{i} \equiv 0$, and the output bits $y_{i}$ yield the digital output $y$ directly in binary format. A nominal value for $\gamma_{i}$ strictly less than two provides fault-tolerant analog implementation, allowing a margin for random variations in $\gamma_{i}, \delta_{i}$ and $\epsilon_{i}$ caused by analog gain and offset errors and corrected through digital calibration [3]. Inverting the recursion (7) backwards from $i=n \ldots 1$ yields a digital output

$$
y=\sum_{i=1}^{n} a_{i} y_{i}+b
$$

which effectively eliminates the quantization noise from all stages $i$ except the very last:

$$
y \equiv x-c_{n} x_{n}
$$

valid for the choice of coefficients

$$
\begin{aligned}
a_{i} & =c_{i} \delta_{i} \\
b & =\sum_{j=1}^{n} c_{j} \epsilon_{j}
\end{aligned}
$$

where $c_{i} \equiv 1 / \prod_{k=1}^{i} \gamma_{k}$. As $\left|x_{n}\right|<1$, the integral error of quantization (9) is bounded by $c_{n}$, supporting linearity at the $n^{\prime}=$ $\sum_{i=1}^{n} \log _{2}\left(\gamma_{i}\right)$ bit level. An efficient (but off-line) incremental auto-calibration procedure to obtain the coefficients (10) in absence of a precise analog reference, is demonstrated in [3].

\subsection{Numerical Example}

A second-order delta-sigma modulator of the topology in Figure 2, and an algorithmic A/D converter with $n=16$ stages and radix $\gamma=$ 1.85 , are used in the behavioral simulations. The analog parameter ranges correspond to typical switched-capacitor implementations as follows: $\alpha_{i}=0.5 \pm 0.01 ; \beta_{i}=0.9975 \pm 0.0025 ; \gamma_{i}=\gamma \pm 0.01$; $\delta_{i}=0.5 \gamma_{i}$ and $\epsilon_{i}= \pm 0.05$. Noise is included in the above models but omitted here for clarity, since it detracts from evaluating the limits of raw SQNR performance attained by calibration alone.

\section{SUPER-NYQUIST BLIND CALIBRATION}

With neither control over a reference nor information on the input signal $x$ other than through the output bits $y_{i}$, the task of estimating the parameters $a_{i}$ and $b$ in (8) from (9) is ill-defined, at best. The approach proposed here is to require a bandlimited input spectrum, with sampling frequency $f_{s}$ strictly above the Nyquist rate $f_{N}=2 f_{c}$. The stop band $\left[f_{c}, f_{s}-f_{c}\right]$ is reserved for calibration purposes, illustrated in Figure 1. Since calibration can be slow, only a negligible overhead in the sampling bandwidth needs to be expended, $f_{s} \approx f_{N}$. No additional cost is incurred since an anti-alias bandlimiting filter is already required for perfect reconstruction of the input.

A high-pass filter $\mathcal{H}_{c}$ spanning the band $\left[f_{c}, f_{s}-f_{c}\right]$ eliminates the input signal $x$. Values of the coefficients $a_{i}$ are then estimated by minimizing the variance of the filtered quantization noise $e=$ $y-x$ :

$$
\left(\mathcal{H}_{c} e\right)^{2}=\left(\sum_{i=1}^{n} a_{i} \mathcal{H}_{c} y_{i}\right)^{2}
$$

solved through a standard least-squares linear regression technique, after eliminating one extra degree of freedom by fixing one of the coefficients $a_{i}\left(a_{1} \equiv 1\right)$. The extra degree of freedom in the scaling of the output is inevitable, since no reference is available to determine the absolute input scale. Similarly, the offset constant $b$ is undefined, eliminated along with $x$ by $\mathcal{H}_{c}$.

An interesting observation arises from comparing the leastsquares $\left(L_{2}\right.$-norm) formulation of the integral nonlinearity in the minimization of (11), with the $L_{\infty}$-norm optimal measure of integral nonlinearity in (9) obtained from the ideal choice of coefficients (10). The difference is illustrated in Figure 3, showing integral error distributions obtained from simulating the A/D converter specified above, with ideal coefficients calculated from (10), calibrated coefficients from minimization of (11), and nominal uncalibrated coefficients (obtained from (10) with $\gamma_{i} \leftarrow \gamma, \delta_{i} \leftarrow \gamma / 2$ and $\left.\epsilon_{i} \leftarrow 0\right)$. The histograms are collected from $5 \times 10^{5}$ random input values, and integral nonlinearity is defined as the difference 


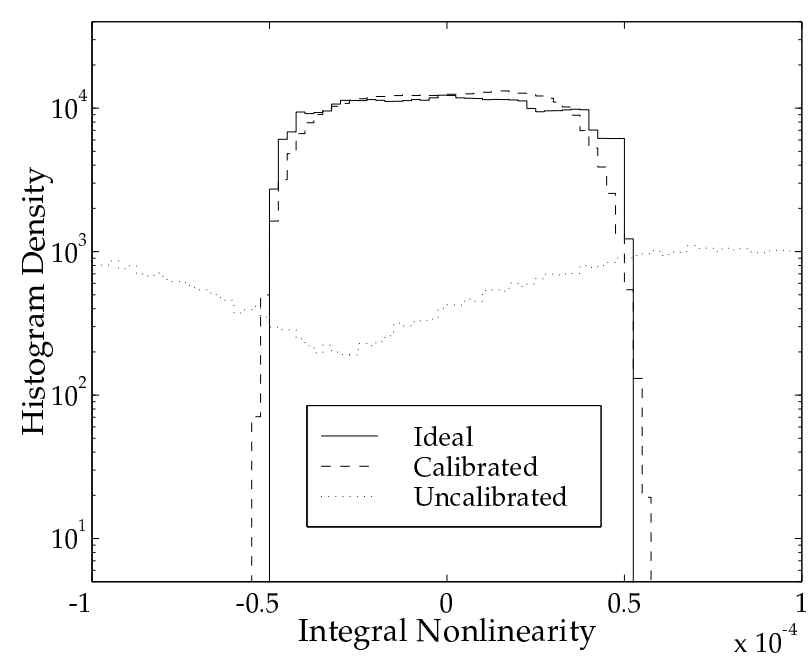

Figure 3: Histogram of algorithmic A/D conversion integral nonlinearity. (-): Ideal coefficients calculated from the analog gain and offset parameters; (- -): Coefficients abtained from blind calibration (4096 points, $\left.f_{s}=2 f_{N}\right) ;(\cdots)$ : Default coefficients from nominal analog gain and offset parameters.

between $x$ and the best linear fit of $y$. It is clear that the ideal calculated coefficient values warrant the lowest worst-case error, but the calibrated values yield lower typical error in the least-squares sense. The $L_{2}$-norm provides a more relevant measure of quantization noise when used in oversampled A/D conversion.

The effect of data size on the quality of the estimated coefficients is illustrated in Figure 4, analyzing the effective resolution of the $\mathrm{A} / \mathrm{D}$ converter obtained by the blind calibration technique as a function of number of calibration samples and relative signal bandwidth $f_{N} / f_{s}$. A lower bound on the number of samples $L$ required for calibration is approximated by

$$
\left(1-\frac{f_{N}}{f_{s}}\right) L>n-1
$$

stating that the rank of the data matrix (effective number of linearly independent calibration samples) needs to exceed the number of estimated parameters $a_{i}$. This approximation agrees with the observations in Figure 4, showing thresholds in calibration length linearly increasing with relative signal bandwidth $f_{N} / f_{s}$. The results indicate excellent performance obtained with just 4,096 calibration samples for a mere $2 \%$ overhead in sampling bandwidth above the Nyquist limit.

\section{NOISE-SHAPING BLIND CALIBRATION}

The super-Nyquist blind calibration technique extends directly to oversampling converters containing multiple stages of delta-sigma modulators and multi-bit quantizers. A fringe benefit of the oversampling is a significant frequency margin for calibration over the entire noise band, $f_{N} / f_{s}=1 /$ OSR where OSR is the oversampling ratio. The only complication arises from the frequency dependency of the quantization error in (5) through the noise shaping. The key to blind calibration here is to match the noise-shaping of

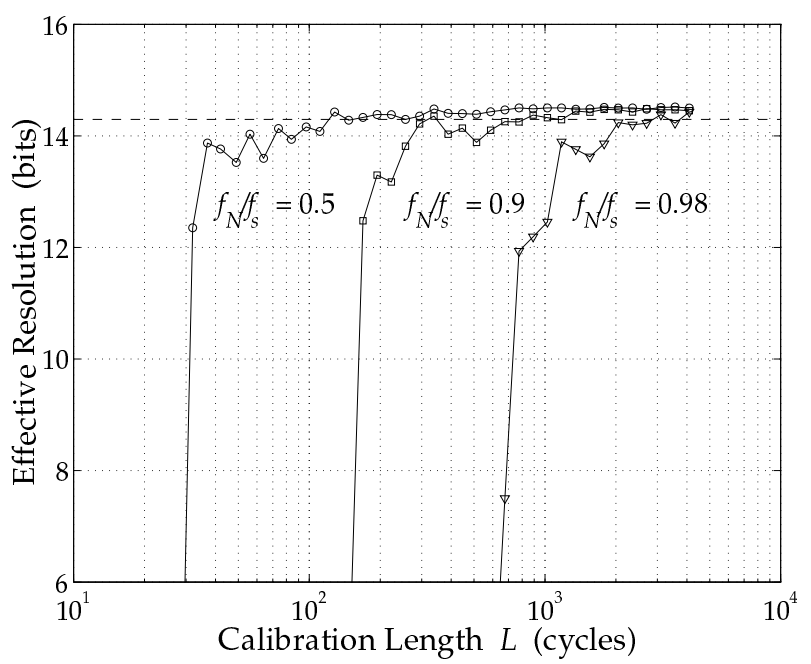

Figure 4: Effective resolution of algorithmic $A / D$ conversion obtained by blind on-line calibration of variable length, for a Nyquist signal bandwidth covering 50\%, 90\% and 98\% of the sampling rate.

the quantization noise as faithfully as possible in the least-squares formulation of the parameter estimates.

By means of example, we consider the Leslie-Singh [5] topology of Figure 2, with second quantizer $Q_{2}$ (3) implemented by the pipelined A/D converter $(9)\left(w \equiv x ; q_{2} \equiv y\right)$. As before, applying the highpass filter $\mathcal{H}_{c}$ to (5) eliminates the bandlimited input $u$ in (5). From (4) and (5), the set of coefficients $a_{i}$ and FIR filters $F_{1}(z)$ and $F_{2}(z)$ are estimated by minimizing the variance of the quantization noise $e_{2}$, assumed white with uniform power spectrum over the calibration band:

$$
\begin{aligned}
\left|\mathcal{H}_{c} e_{2}\right|^{2} & =\left|\frac{\alpha_{1}}{z-\beta_{1}} \frac{\alpha_{2}}{z-\beta_{2}} \mathcal{H}_{c} q\right|^{2} \\
& \approx\left|F_{1}(z) \mathcal{N}_{c} q_{1}+F_{2}(z) \mathcal{N}_{c} q_{2}\right|^{2} \\
& =\left|F_{1}(z) \mathcal{N}_{c} q_{1}+F_{2}(z) \sum_{i=1}^{n} a_{i} \mathcal{N}_{c} y_{i}\right|^{2}
\end{aligned}
$$

where $z \equiv e^{j \Omega}$ with $\Omega=2 \pi f / f_{s}$, and the digital emphasis filter

$$
\mathcal{N}_{c}(z)=\frac{4}{(z-1)^{2}} \mathcal{H}_{c}(z) \approx \frac{\alpha_{1}}{z-\beta_{1}} \frac{\alpha_{2}}{z-\beta_{2}} \mathcal{H}_{c}(z)
$$

serves to equalize the second-order noise-shaping of the spectrum of $e_{2}$ in the estimation, besides removing the signal band of $u$. The error made in the approximation of the unknown noise-shaping in (14) does not affect the accuracy of the results to first order.

The solution consists of minimizing (13) (in time or frequency domain), while fixing one of the coefficients to eliminate one degree of freedom as before. The least-squares estimation is nonlinear in the parameters, since the coefficients in $F_{2}(z)$ and $a_{i}$ appear together in a product. A simple iterative means to linearize the estimation is to alternatingly solve for the $F_{1}(z)$ and $a_{i}$ coefficients while fixing $F_{2}(z)$ (plus one other coefficient), and subsequently solving for $F_{1}(z)$ and $F_{2}(z)$ fixing the $a_{i}$ coefficients (plus one 


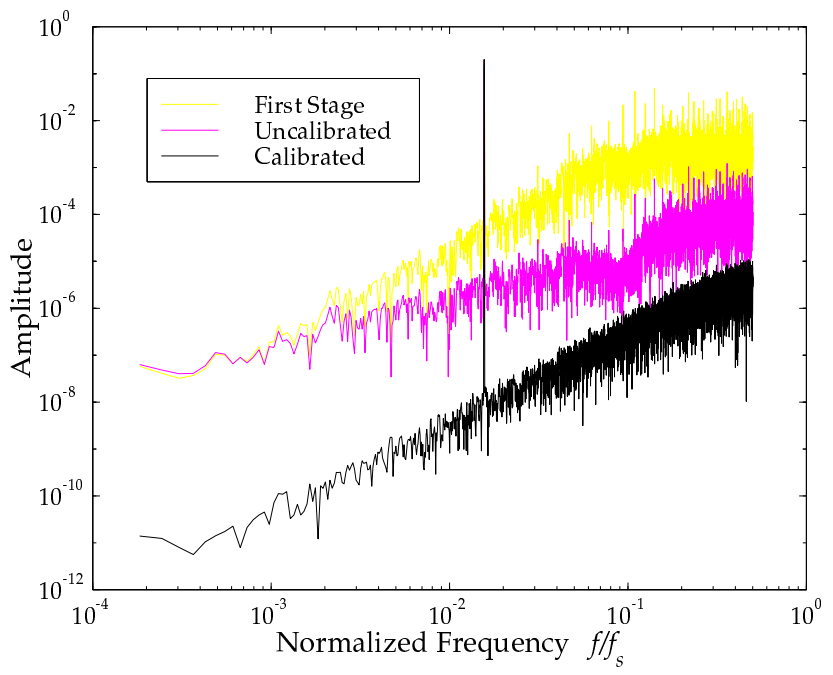

Figure 5: Output amplitude spectrum in response to a sinusoidal input. Top: Single-bit second-order $\Delta \Sigma$ modulator output $q_{1}$ from the first stage. Center: Combined dual-quantization output y from $\Delta \Sigma$ modulator and 16-stage radix-1.85 algorithmic $A / D$ converter, with nominal, uncalibrated coefficients. Bottom: Calibrated output $y$.

other). From nominal initial settings for $F_{2}(z)$, this procedure converges in about 5 iteration cycles in our numerical experiments.

The simulation results demonstrate significant improvements in signal to quantization noise ratio (SQNR) achieved over the uncalibrated case, in which default values for the A/D coefficients and $\Delta \Sigma$ filters are assigned according to nominal values of the analog circuit parameters. As evident from the modulation spectrum in response to a harmonic input in Figure 5, the improvements result from a combination of eliminating dominant first-order quantization noise from the first stage, and linearizing the pipelined A/D multi-bit quantizer. By forcing a flat spectrum for $e_{2}$ in (13), any harmonic distortion present in the $q_{1}$ output due to strong correlations between $w$ and $u$ is eliminated.

The dependence of SQNR on oversampling ratio is shown in 6. The blind calibration is most successful at higher oversampling ratios where the calibration band covers a larger portion of the noise-shaped spectrum, although improvements at low oversampling ratios are still significant, within $10 \mathrm{~dB}$ of the ideal case. The astronomical figures of SQNR are only to illustrate the correction power of the calibration procedure, which clearly goes beyond practical limits that are physically attainable with noise levels present in typical analog circuits. To validate robustness of the above calibration techniques under more general conditions, we have conducted simulations including additive noise (in (1), (7) and the argument of (2)), showing no significant degradations in SNR performance obtained from noisy calibration compared to that of the ideal coefficients under identical noisy conditions.

\section{CONCLUSIONS}

We have shown that under the mild assumption of a bandlimited input signal, on-line digital calibration of analog linear variability in the implementation of Nyquist and oversampled converters is

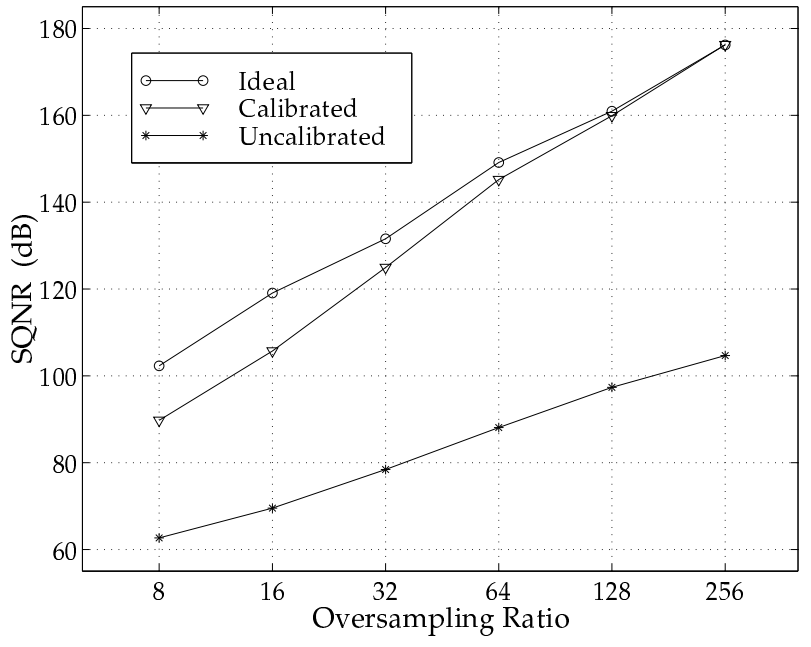

Figure 6: Signal to quantization noise ratio (SQNR) as a function of oversampling ratio for the dual-quantization modulator, with ideal coefficients, and with calibrated coefficients obtained at maximum calibration bandwidth supported by the oversampling ratio.

achieved using no more information than direct observations of the digital outputs being calibrated, with no need to apply a signal reference or interrupting the data conversion process. The procedure is widely applicable to multi-stage designs, and was verified with behavioral simulations on pipelined algorithmic and Leslie-Singh dual-stage multi-bit oversampled A/D converters, with near-perfect correction at virtually no expense in signal bandwidth, and SQNR improvements beyond physical accuracy limits in analog circuit implementations.

\section{REFERENCES}

[1] P.R. Gray, B.A. Wooley, and R.W. Brodersen, Eds., Analog MOS Integrated Circuits, II, NJ: IEEE Press, 1988.

[2] J.C. Candy and G.C. Temes, "Oversampled Methods for A/D and D/A Conversion," in Oversampled Delta-Sigma Data Converters, IEEE Press, pp 1-29, 1992.

[3] A.N. Karanicolas, H.-S. Lee and K.L. Bacrania, "A 15-b 1-Msample/s Digitally Self-Calibrated Pipeline ADC," IEEE J. Solid-State Circuits, vol. 28 (12), pp 1207-1215, 1993.

[4] T. Hayashi, Y. Inabe, K. Uchimura, T. Kimura, "A Multistage DeltaSigma Modulator without Double Integration Loop," ISSCC Tech. Dig. Pap., vol. 39, pp 182-183, 1986.

[5] T.C. Leslie and B. Singh, "An Improved Sigma-Delta Modulator Architecture," Proc. IEEE Int. Symp. Circuits and Systems (ISCAS'90), pp 372-375, 1990.

[6] S. Abdennadher, S. Kiaei, G.C. Temes, and R. Schreier, "Adaptive Self-Calibrating Delta-Sigma Modulators," Electronics Letters, vol. 28 (14), pp 1288-1289, 1992.

[7] G. Cauwenberghs and G.C. Temes, "Adaptive Calibration of Multiple Quantization Oversampled A/D Converters," Proc. IEEE International Symposium on Circuits and Systems (ISCAS'96), Atlanta GA, 1996, vol. I, pp 512-515.

[8] A. Wiesbauer and G.C. Temes, "Adaptive Digital Compensation of Analog Circuit Imperfections for Cascaded $\Delta \Sigma$ Modulators," Proc. 30th Asilomar Conf. on Signals, Systems and Computers, Pacific Grove CA, Nov. 3-6, 1996. 